Kansas State University Libraries

New Prairie Press

\title{
COMPARING ANALYSES OF UNBALANCED SPLIT-PLOT EXPERIMENTS
}

Christina D. Smith

Dallas E. Johnson

Follow this and additional works at: https://newprairiepress.org/agstatconference

Part of the Agriculture Commons, and the Applied Statistics Commons

\section{c) (i) $९$}

This work is licensed under a Creative Commons Attribution-Noncommercial-No Derivative Works 4.0 License.

\section{Recommended Citation}

Smith, Christina D. and Johnson, Dallas E. (2004). "COMPARING ANALYSES OF UNBALANCED SPLIT-PLOT EXPERIMENTS," Conference on Applied Statistics in Agriculture. https://doi.org/10.4148/2475-7772.1161

This is brought to you for free and open access by the Conferences at New Prairie Press. It has been accepted for inclusion in Conference on Applied Statistics in Agriculture by an authorized administrator of New Prairie Press. For more information, please contact cads@k-state.edu. 


\title{
COMPARING ANALYSES OF UNBALANCED SPLIT-PLOT EXPERIMENTS
}

\author{
Christina D. Smith \\ Kansas State University Kansas State University
}

\begin{abstract}
Several procedures for constructing confidence intervals and testing hypotheses about fixed effects in unbalanced split-plot experiments have previously been presented and discussed by Remmenga and Johnson. They recommended a few of the procedures they considered as useful and reliable procedures. Since the advent of the SAS $^{\circledR}$ MIXED procedure, mixed model analyses with REML estimates of the variance components are easily accessible to researchers. This paper compares the analysis of unbalanced split-plot experiments using mixed model procedures with REML estimates of the variance components to the previously established procedures by means of additional simulation studies.
\end{abstract}

Keywords: unbalanced, split-plots, mixed models, variance components, REML

\section{INTRODUCTION}

This paper discusses four methods for constructing confidence intervals and hypothesis tests about fixed effects for unbalanced split-plot experiments. It considers three methods recommended by Remmenga and Johnson (1995) and one additional method. All of the procedures discussed can be applied to repeated measures experiments when the variancecovariance matrix satisfies the Huynh-Feldt (1970) conditions.

Consider a split-plot experiment with two treatments $a$ and $b$ where $a$ is the whole plot treatment and $b$ is the subplot treatment. When the whole plot treatments are randomly assigned to the whole plot experimental units, a model for such an experiment is

$$
y_{i j k}=\mu+\alpha_{i}+\delta_{k(i)}+\beta_{j}+(\alpha \beta)_{\mathrm{ij}}+\varepsilon_{i j k}
$$

for $i=1,2, \ldots, a, j=1,2, \ldots, b$ and $k=1,2, \ldots, n_{i}$, where $\mu$ is an overall mean, $\alpha_{i}$ is the $i^{t h}$ whole plot treatment effect, $\delta_{k(i)} \sim N\left(0, \sigma_{\delta}{ }^{2}\right)$ is the whole plot error, $\beta_{j}$ is the $j^{\text {th }}$ subplot treatment effect, $(\alpha \beta)_{i j}$ is the interaction between the $i^{\text {th }}$ whole plot treatment, the $j^{\text {th }}$ subplot treatment, and

$\varepsilon_{i j k} \sim N\left(0, \sigma_{\varepsilon}{ }^{2}\right)$ is the subplot error. Also, note that, $k(i)$ identifies each subject within a whole plot unit and $n_{i}$ is the number of whole plot experimental units assigned to each of the whole plot treatments, $i=1,2, \ldots, a$. In addition all of the $\delta_{k(i)}$ 's and the $\varepsilon_{i j k}$ 's are assumed to be independent.

This model can also be expressed as a mixed model that has the form

$$
\mathbf{y}=\mathbf{X} \boldsymbol{\beta}+\mathbf{Z} \boldsymbol{\delta}+\boldsymbol{\varepsilon}
$$

where $\boldsymbol{\delta} \sim \mathrm{N}_{\mathrm{n}}\left(\mathbf{0}, \sigma_{\delta}{ }^{2} \mathbf{I}\right)$ and $\boldsymbol{\varepsilon} \sim \mathrm{N}_{\mathrm{N}}\left(\mathbf{0}, \sigma_{\varepsilon}{ }^{2} \mathbf{I}\right),{\sigma_{\delta}}^{2}$ and ${\sigma_{\varepsilon}}^{2}$ are not known and $\boldsymbol{\delta}$ and $\boldsymbol{\varepsilon}$ are distributed independently of each other. In addition, $\mathbf{X}$ is a known $N \times p$ design matrix 
corresponding to the fixed effects, $\mathbf{Z}$ is a known $N \times n$ design matrix corresponding to the random effects, where $n=\sum_{i=1}^{a} n_{i}$, and $\mathrm{N}$ is the total number of observations and $\boldsymbol{\beta}$ is a $p \times 1$ vector of unknown parameters where $p=(a+1)(b+1)$ is the total number of fixed effects parameters.

Now, when an experiment is unbalanced in the subplots, the $n_{i}$ 's may not be equal and $y_{i j k}$ may not be observed for every $j$ within each possible $i$ and $k$ combination. This paper assumes that at least one $y_{i j k}$ is observed for every $i=1,2, \ldots, a$ and $k=1,2, \ldots, n_{i}$, that is, there is at least one observation for every experimental unit assigned to a whole plot treatment. It is also assumed that $\mu+\alpha_{i}+\beta_{j}+(\alpha \beta)_{\mathrm{ij}}$ is estimable for every $i$ and $j$. That is, $\mu+\alpha_{i}+\beta_{j}+(\alpha \beta)_{\mathrm{ij}}$, $i=1,2, \ldots, a$, and $j=1,2, \ldots, b$ is a basis set of estimable functions of the parameters, $\mu, \alpha_{1}, \ldots, \alpha_{a}, \beta_{1}, \ldots, \beta_{b},(\alpha \beta)_{11}, \ldots,(\alpha \beta)_{\mathrm{ab}}$. Thus confidence intervals and hypothesis tests about linear combinations of the elements in the basis set can be constructed. It might also be noted that $\mu+\alpha_{i}+\beta_{j}+(\alpha \beta)_{\mathrm{ij}}$ is estimable if there is at least one observed $y_{i j k}$ for each $(i, j)$ combination.

\section{PROCEDURES}

The procedures discussed by Remmenga and Johnson (1995) considered fourteen procedures for the analyses of fixed effects for unbalanced split-plot experiments. They recommended three of the procedures that they considered, two of which initially treated the random effects as fixed. The third procedure recommended by Remmenga and Johnson (1995) is a mixed model procedure with uses method of moments estimates for the variance components. The purpose of this paper is to compare the procedure used by PROC MIXED in $\mathrm{SAS}^{\circledR}$ Version 8.2 with its METHOD=REML default to the procedures recommended by Remmenga and Johnson (1995). All of the procedures considered in this paper are for constructing confidence intervals and hypothesis tests about $\boldsymbol{I}^{\prime} \boldsymbol{\beta}$ which is assumed to be an estimable linear combination of the elements in the vector $\boldsymbol{\beta}$. The primary complication in accomplishing this task is that $\sigma_{\delta}{ }^{2}$ and $\sigma_{\varepsilon}{ }^{2}$ are not known and must be estimated. Each of the four methods deal with different ways to estimate $\sigma_{\delta}^{2}$ and $\sigma_{\varepsilon}^{2}$.

2.1 PROCEDURE 1 - Milliken and Johnson

The first procedure recommended by Remmenga and Johnson was originally proposed by Milliken and Johnson (1984). The model $\mathbf{y}=\mathbf{X} \boldsymbol{\beta}+\mathbf{Z} \boldsymbol{\delta}+\boldsymbol{\varepsilon}$ can be written as $\mathbf{y}=\mathbf{X} * \boldsymbol{\beta} *+\boldsymbol{\varepsilon}$ where $\mathbf{X}^{*}=[\mathbf{X} \mathbf{Z}], \boldsymbol{\beta}^{*}=\left[\begin{array}{l}\boldsymbol{\beta} \\ \boldsymbol{\delta}\end{array}\right]$ and $\boldsymbol{\varepsilon} \sim \mathrm{N}_{\mathrm{N}}\left(\mathbf{0}, \sigma_{\varepsilon}{ }^{2} \mathbf{I}\right)$. Then one solution to the normal equations, $\mathbf{X}^{* \prime} \mathbf{X}^{*} \boldsymbol{\beta}^{*}=\mathbf{X}^{* \prime} \mathbf{y}$, is $\hat{\boldsymbol{\beta}}^{*}=\left[\begin{array}{l}\hat{\boldsymbol{\beta}} \\ \hat{\boldsymbol{\delta}}\end{array}\right]=\mathbf{X}^{*^{-}} \mathbf{y}$, where $\mathbf{X}^{*^{-}}$is the Moore-Penrose generalized inverse 
of $\mathbf{X}^{*}$. If $\boldsymbol{I}$ is in the column space of $\mathbf{X}$ and $\mathbf{b}$ is chosen so that $\left[\begin{array}{l}\boldsymbol{I} \\ \mathbf{b}\end{array}\right]$ is in the column space of $\mathbf{X}^{* \prime}$, then

$$
\left[\begin{array}{l}
\boldsymbol{l} \\
\mathbf{b}
\end{array}\right]^{\prime} \hat{\boldsymbol{\beta}}^{*}=\boldsymbol{l}^{\prime} \hat{\boldsymbol{\beta}}+\mathbf{b}^{\prime} \hat{\boldsymbol{\delta}}
$$

is an unbiased estimator of $\boldsymbol{I}^{\prime} \boldsymbol{\beta}$. The elements in $\mathbf{b}$ are usually chosen so that they treat the $\delta_{k(i)}$ equally for every $k$ within each $i$. That is, in the vector $\mathbf{b}^{\prime} \hat{\boldsymbol{\delta}}$ each of the $\delta_{k(1)}$ receive equal weights, while each of the $\delta_{k(2)}$ receive equal weights, and so on.

The variance of $\boldsymbol{I}^{\prime} \hat{\boldsymbol{\beta}}+\mathbf{b}^{\prime} \hat{\boldsymbol{\delta}}$ is given by

$$
\operatorname{var}\left[\boldsymbol{I}^{\prime} \hat{\boldsymbol{\beta}}+\mathbf{b}^{\prime} \hat{\boldsymbol{\delta}}\right]=u \sigma_{\varepsilon}^{2}+w \sigma_{\delta}^{2}=u\left(\sigma_{\varepsilon}^{2}+\frac{w}{u} \sigma_{\delta}^{2}\right)
$$

where

$$
w=\left[\begin{array}{l}
\boldsymbol{l} \\
\mathbf{b}
\end{array}\right]^{\prime} \mathbf{X}^{*^{-}} \mathbf{Z} \mathbf{Z}^{\prime} \mathbf{X}^{*^{-}-}\left[\begin{array}{l}
\boldsymbol{l} \\
\mathbf{b}
\end{array}\right]=\left[\begin{array}{l}
\boldsymbol{l} \\
\mathbf{b}
\end{array}\right]^{\prime}\left[\begin{array}{ll}
\mathbf{X} & \mathbf{Z}
\end{array}\right]^{-} \mathbf{Z Z} \mathbf{Z}^{\prime}\left[\begin{array}{l}
\mathbf{X}^{\prime} \\
\mathbf{Z}^{\prime}
\end{array}\right]^{-}\left[\begin{array}{l}
\boldsymbol{l} \\
\mathbf{b}
\end{array}\right]
$$

and

$$
u=\left[\begin{array}{l}
\boldsymbol{I} \\
\mathbf{b}
\end{array}\right]^{\prime} \mathbf{X}^{*^{-}} \mathbf{X}^{*^{\prime}}\left[\begin{array}{l}
\boldsymbol{I} \\
\mathbf{b}
\end{array}\right]=\left[\begin{array}{l}
\boldsymbol{I} \\
\mathbf{b}
\end{array}\right]^{\prime}\left[\begin{array}{ll}
\mathbf{X} & \mathbf{Z}
\end{array}\right]^{-}\left[\begin{array}{l}
\mathbf{X}^{\prime} \\
\mathbf{Z}^{\prime}
\end{array}\right]\left[\begin{array}{l}
\boldsymbol{I} \\
\mathbf{b}
\end{array}\right]
$$

As previously noted $\sigma_{\delta}{ }^{2}$ and $\sigma_{\varepsilon}^{2}$ are not known and must be estimated in order to estimate the variance of $\boldsymbol{I}^{\prime} \hat{\boldsymbol{\beta}}+\mathbf{b}^{\prime} \hat{\boldsymbol{\delta}}$. Let $s_{1}^{2}$ be the whole plot error mean square with $v_{1}$ degrees of freedom and expected value $\sigma_{\varepsilon}^{2}+q \sigma_{\delta}^{2}$ where

$$
q=\frac{\operatorname{Tr}\left[\left(\mathbf{X}^{*} \mathbf{X}^{*^{-}}-\mathbf{X} \mathbf{X}^{-}\right) \mathbf{Z} \mathbf{Z}^{\prime}\right]}{n-a}
$$

and let $s_{2}^{2}$ be the subplot error mean square with $v_{2}$ degrees of freedom and expectation $\sigma_{\varepsilon}^{2}$. Then method of moments estimators of $\sigma_{\delta}^{2}$ and $\sigma_{\varepsilon}^{2}$ are $\hat{\sigma}_{\varepsilon}^{2}=s_{2}^{2}$ and $\hat{\sigma}_{\delta}^{2}=\frac{s_{1}^{2}-s_{2}^{2}}{q}$. An unbiased estimate of $\operatorname{var}\left[\boldsymbol{l}^{\prime} \hat{\boldsymbol{\beta}}+\mathbf{b}^{\prime} \hat{\boldsymbol{\delta}}\right]$ is

$$
\operatorname{vâr}\left[\boldsymbol{l}^{\prime} \hat{\boldsymbol{\beta}}+\mathbf{b}^{\prime} \hat{\boldsymbol{\delta}}\right]=u\left(\hat{\sigma}_{\varepsilon}^{2}+\frac{w}{u} \hat{\sigma}_{\delta}^{2}\right)=\frac{1}{q}\left\{w s_{1}^{2}+(u q-w) \mathrm{s}_{2}^{2}\right\}
$$


Note that when $s_{2}^{2}$ is greater than $s_{1}^{2}, \hat{\sigma}_{\delta}^{2}$ could be negative. For such occurrences, Remmenga and Johnson recommended replacing $\hat{\sigma}_{\delta}^{2}$ with zero so that $\operatorname{var}\left[\boldsymbol{l}^{\prime} \hat{\boldsymbol{\beta}}+\mathbf{b}^{\prime} \hat{\boldsymbol{\delta}}\right]=u \hat{\sigma}_{\delta}^{2}=u s_{2}^{2}$.

$$
\text { Now, } \frac{\left(v^{*}\right) \operatorname{var}\left[\boldsymbol{I}^{\prime} \hat{\boldsymbol{\beta}}+\mathbf{b}^{\prime} \hat{\boldsymbol{\delta}}\right]}{\operatorname{var}\left[\boldsymbol{I}^{\prime} \hat{\boldsymbol{\beta}}+\mathbf{b}^{\prime} \hat{\boldsymbol{\delta}}\right]} \dot{\sim} \chi_{v^{*}}^{2}, \text { where } v^{*} \text { is estimated using the Satterthwaite }
$$

procedure. That is, the estimate of $v^{*}$ is

if $s_{1}^{2}>s_{2}^{2}$ and

$$
\hat{v}^{*}=\frac{\left[w s_{1}^{2}+(u q-w) \mathrm{s}_{2}^{2}\right]^{2}}{\frac{\left[w s_{1}^{2}\right]^{2}}{v_{1}}+\frac{\left[(u q-w) s_{2}^{2}\right]^{2}}{v_{2}}}
$$

$$
\hat{v}^{*}=v_{2}
$$

if $s_{1}^{2} \leq s_{2}^{2}$.

Thus an approximate $(1-\alpha) 100 \%$ confidence interval about $\boldsymbol{I}^{\prime} \boldsymbol{\beta}$ is

$$
\boldsymbol{I}^{\prime} \hat{\boldsymbol{\beta}}+\mathbf{b}^{\prime} \hat{\boldsymbol{\delta}} \pm t_{\alpha / 2, \hat{v}^{*}} \sqrt{\operatorname{vâr}\left[\boldsymbol{I}^{\prime} \hat{\boldsymbol{\beta}}+\mathbf{b}^{\prime} \hat{\boldsymbol{\delta}}\right]}
$$

and the test statistic to test $\boldsymbol{I}^{\prime} \hat{\boldsymbol{\beta}}=0$ is $t=\frac{\boldsymbol{I}^{\prime} \hat{\boldsymbol{\beta}}+\mathbf{b}^{\prime} \hat{\boldsymbol{\delta}}}{\sqrt{\operatorname{var}\left[\boldsymbol{I}^{\prime} \hat{\boldsymbol{\beta}}+\mathbf{b}^{\prime} \hat{\boldsymbol{\delta}}\right]}}$. The degrees of freedom associated with this approximate $t$ statistic are estimated by the Satterthwaite method as $\hat{v}^{*}$.

\subsection{PROCEDURE 2 - Minimal Sufficient Statistics}

Remmenga and Johnson point out that for Procedure 1 the distribution of $s_{1}^{2}$ may not always be proportional to a chi-square distribution and may not be distributed independently of $\left[\begin{array}{l}\boldsymbol{l} \\ \mathbf{b}\end{array}\right]^{\prime} \hat{\boldsymbol{\beta}}^{*}$. Consequently, they considered using a procedure described by Hultquist and Atzinger (1972) to obtain a set of mutually independent unbiased minimal sufficient statistics for an unbalanced split-plot design using a nonsingular transformation of the observation vector, $\mathbf{y}$. The procedure is rather cumbersome and will not be described here, however, it was one of the three procedures they recommended. See Remmenga and Johnson for details.

2.3 PROCEDURE 3 - Mixed Models with Method of Moments Estimators

The third procedure recommended by Remmenga and Johnson is a mixed model method that uses method of moments estimators of $\sigma_{\varepsilon}{ }^{2}$ and $\sigma_{\delta}{ }^{2}$. First, note that the model can be written as $\mathbf{y}=\mathbf{X} \boldsymbol{\beta}+\boldsymbol{\varepsilon}$ where $\boldsymbol{\varepsilon} \sim N_{\mathrm{N}}(\mathbf{0}, \boldsymbol{\Sigma})$. When

$$
\boldsymbol{\Sigma}={\sigma_{\varepsilon}}^{2} \mathbf{I}+\mathbf{Z} \operatorname{cov}(\delta) \mathbf{Z}^{\prime}={\sigma_{\varepsilon}}^{2} \mathbf{I}+\mathbf{Z}\left(\sigma_{\delta}^{2} \mathbf{I}\right) \mathbf{Z}^{\prime}={\sigma_{\varepsilon}}^{2} \mathbf{I}+{\sigma_{\delta}}^{2} \mathbf{Z} \mathbf{Z}^{\prime}
$$


is known, the normal equations are $\left[\mathbf{X}^{\prime} \boldsymbol{\Sigma}^{-1} \mathbf{X}\right] \boldsymbol{\beta}=\mathbf{X}^{\prime} \boldsymbol{\Sigma}^{-1} \mathbf{y}$ and a solution is given by $\hat{\boldsymbol{\beta}}=\left[\mathbf{X}^{\prime} \boldsymbol{\Sigma}^{-1} \mathbf{X}\right]^{-} \mathbf{X}^{\prime} \boldsymbol{\Sigma}^{-1} \mathbf{y}$. Then the UMVU estimator of $\boldsymbol{I}^{\prime} \boldsymbol{\beta}$ is $\boldsymbol{I}^{\prime} \hat{\boldsymbol{\beta}}$ and $\operatorname{var}\left(\boldsymbol{I}^{\prime} \hat{\boldsymbol{\beta}}\right)=\boldsymbol{I}^{\prime}\left[\mathbf{X}^{\prime} \boldsymbol{\Sigma}^{-1} \mathbf{X}\right]^{-} \boldsymbol{I}$. As before, $\sigma_{\varepsilon}{ }^{2}$ and $\sigma_{\delta}{ }^{2}$ are unknown and must be estimated. The method of moments estimators of ${\sigma_{\varepsilon}}^{2}$ and $\sigma_{\delta}{ }^{2}$ are $\hat{\sigma}_{\varepsilon}^{2}=s_{2}^{2}$ and $\hat{\sigma}_{\delta}^{2}=\frac{s_{1}^{2}-s_{2}^{2}}{q}$, respectively, where $s_{1}^{2}, s_{2}^{2}$, and $q$ are as given in Procedure 1. The variance-covariance matrix $\Sigma$ is estimated by $\hat{\boldsymbol{\Sigma}}=s_{2}^{2} \mathbf{I}+\left(\frac{s_{1}^{2}-s_{2}^{2}}{q}\right) \mathbf{Z Z} \mathbf{Z}^{\prime}$ if $s_{1}^{2}>s_{2}^{2}$ and by $\hat{\boldsymbol{\Sigma}}=s_{2}^{2} \mathbf{I}$ if $s_{1}^{2} \leq s_{2}^{2}$. Then $\boldsymbol{I}^{\prime} \boldsymbol{\beta}$ is estimated by $\boldsymbol{I}^{\prime} \hat{\boldsymbol{\beta}}_{M O M}$ where $\hat{\boldsymbol{\beta}}_{M O M}=\left[\mathbf{X}^{\prime} \hat{\boldsymbol{\Sigma}}^{-1} \mathbf{X}\right]^{-} \mathbf{X}^{\prime} \hat{\boldsymbol{\Sigma}}^{-1} \mathbf{y}$ and the variance of $\boldsymbol{I}^{\prime} \hat{\boldsymbol{\beta}}_{M O M}$ is estimated by $\operatorname{vâr}\left(\boldsymbol{I}^{\prime} \hat{\boldsymbol{\beta}}_{M O M}\right)=\boldsymbol{I}^{\prime}\left[\mathbf{X}^{\prime} \hat{\boldsymbol{\Sigma}}^{-1} \mathbf{X}\right]^{-} \boldsymbol{I}$.

Now, $\frac{\boldsymbol{I}^{\prime} \hat{\boldsymbol{\beta}}_{M O M}-\boldsymbol{I}^{\prime} \boldsymbol{\beta}}{\sqrt{\operatorname{vâr}\left(\boldsymbol{I} \hat{\boldsymbol{\beta}}_{M O M}\right)}}$ is often approximated by a standard normal distribution, but this cannot be recommended for small sample sizes (Remmenga and Johnson, 1995). Remmenga and Johnson follow a recommendation by McLean and Sanders (1988) that $\frac{\boldsymbol{I}^{\prime} \hat{\boldsymbol{\beta}}_{M O M}-\boldsymbol{I}^{\prime} \boldsymbol{\beta}}{\sqrt{\operatorname{var}\left(\boldsymbol{I}^{\prime} \hat{\boldsymbol{\beta}}_{M O M}\right)}}$ be approximated by a student's $t$ distribution with $\hat{v}^{*}$ degrees of freedom, the same degrees of freedom given for Procedure 1. Then an approximate $(1-\alpha) 100 \%$ confidence interval about $\boldsymbol{I}^{\prime} \boldsymbol{\beta}$ is $\boldsymbol{I}^{\prime} \hat{\boldsymbol{\beta}}_{M O M} \pm t_{\alpha / \mathbf{2}, \hat{v}^{*}} \sqrt{\operatorname{vâr}\left[\boldsymbol{I}^{\prime} \hat{\boldsymbol{\beta}}_{M O M}\right]}$.

2.4 PROCEDURE 4 - Mixed Models with REML Estimators

Procedure 4 is also a mixed model procedure except that $\sigma_{\delta}{ }^{2}$ and $\sigma_{\varepsilon}{ }^{2}$ are estimated by the restricted maximum likelihood (REML) approach. The model is still $\mathbf{y}=\mathbf{X} \boldsymbol{\beta}+\mathbf{Z} \boldsymbol{\delta}+\boldsymbol{\varepsilon}$ where $\boldsymbol{\delta} \sim N_{\mathrm{n}}\left(\mathbf{0}, \sigma_{\delta}{ }^{2} \mathbf{I}\right)$ and $\boldsymbol{\varepsilon} \sim N_{\mathrm{N}}\left(\mathbf{0}, \sigma_{\varepsilon}{ }^{2} \mathbf{I}\right)$, and that $\boldsymbol{\delta}$ and $\boldsymbol{\varepsilon}$ are distributed independently of each other. Again $\sigma_{\delta}{ }^{2}$ and $\sigma_{\varepsilon}{ }^{2}$ are not known so they need to be estimated.

Now $\mathbf{X}$ is a known $N \times p$ matrix of rank $k$. Let $\mathbf{L}$ be a $(n-k) \times N$ matrix of rank $n-k$ such that $\mathbf{L X}=0$. Let $\mathbf{y}^{*}=\mathbf{L y}=\mathbf{L Z} \boldsymbol{\delta}+\mathbf{L} \boldsymbol{\varepsilon}$. Note that $\mathbf{y}^{*} \sim \mathrm{N}_{\mathrm{n}-\mathrm{k}}(\mathbf{0}, \mathbf{V})$ where $\mathbf{V}=\sigma_{\delta}^{2} \mathbf{L} \mathbf{Z Z} \mathbf{L}^{\prime}+\sigma_{\varepsilon}^{2} \mathbf{L} \mathbf{L}^{\prime}$. The REML estimates of ${\sigma_{\varepsilon}}^{2}$ and $\sigma_{\delta}{ }^{2}$ are the respective values of $\sigma_{\varepsilon}{ }^{2}$ and $\sigma_{\delta}^{2}$ that maximize the likelihood function

$$
\mathrm{L}\left(\sigma_{\varepsilon}^{2}, \sigma_{\delta}^{2} ; \mathbf{y}^{*}\right)=\frac{1}{(2 \pi)^{\frac{\mathrm{n}-\mathrm{k}}{2}}\left|\sigma_{\delta}^{2} \mathbf{L} \mathbf{Z} \mathbf{Z}^{\prime} \mathbf{L}^{\prime}+\sigma_{\varepsilon}^{2} \mathbf{L} \mathbf{L}^{\prime}\right|^{\frac{\mathrm{n}-\mathrm{k}}{2}}} \exp \left\{\frac{-1}{2} \mathbf{y}^{*^{\prime}}\left(\sigma_{\delta}^{2} \mathbf{L} \mathbf{Z Z} \mathbf{Z}^{\prime}+\sigma_{\varepsilon}^{2} \mathbf{L} \mathbf{L}^{\prime}\right)^{-1} \mathbf{y}^{*}\right\}
$$


Then $\boldsymbol{I}^{\prime} \boldsymbol{\beta}$ is estimated by $\boldsymbol{I}^{\prime} \hat{\boldsymbol{\beta}}_{R E M L}$ where $\hat{\boldsymbol{\beta}}_{R E M L}=\left[\mathbf{X}^{\prime} \hat{\mathbf{V}}^{-1} \mathbf{X}\right]^{-} \mathbf{X}^{\prime} \hat{\mathbf{V}}^{-1} \mathbf{y}$ and $\hat{\mathbf{V}}=\hat{\sigma}_{\delta}^{2} \mathbf{L} \mathbf{Z Z} \mathbf{Z}^{\prime}+\hat{\sigma}_{\varepsilon}^{2} \mathbf{L} \mathbf{L}^{\prime}$. The variance of $\boldsymbol{I}^{\prime} \hat{\boldsymbol{\beta}}_{R E M L}$ is often simply estimated by $\operatorname{vâr}\left(\boldsymbol{I}^{\prime} \hat{\boldsymbol{\beta}}_{R E M L}\right)=\boldsymbol{I}^{\prime}\left[\mathbf{X}^{\prime} \hat{\mathbf{V}}^{-1} \mathbf{X}\right]^{-} \boldsymbol{I}$. However, Kenward and Roger (1997) give a different approximation since the expression above underestimates the true variance of $\boldsymbol{I} \hat{\boldsymbol{\beta}}_{R E M L}$ as shown by Kackar and Harville (1984). The simulations discussed in the next section use Kenward and Roger's recommendations.

\section{SIMULATION STUDY}

A set of simulations was conducted so that comparisons between the four different procedures could be made. Remmenga (1992) conducted simulations for the three procedures recommended by Remmenga and Johnson as well as for several other procedures. However, she did no simulations using REML estimates of the variance components. Her results are combined with the results of an additional set of simulations in order to make comparisons between the previously recommended procedures and the mixed model procedure using REML estimates of the variance components.

The general model for all of the simulations is $y_{i j k}=\mu_{i j}+\delta_{k(i)}+\varepsilon_{i j k}$ where $\mu_{i j}=\mu+\alpha_{i}+\beta_{j}+(\alpha \beta)_{i j}$ for $i=1,2, \ldots, \mathrm{a}, j=1,2, \ldots, \mathrm{b}$ and $k=1,2, \ldots \mathrm{n}_{i}$. The values for $\delta_{k(i)}$ and $\varepsilon_{i j k}$ were randomly generated from independent normal distributions with means equal to zero and variances $\sigma_{\delta}^{2}$ and $\sigma_{\varepsilon}{ }^{2}$, respectively. This paper considers the unbalanced split-plot where the variance of the whole plot varies relative to the variance of the subplot, as was done by Remmenga and Johnson. Thus, without loss of generality, $\sigma_{\varepsilon}{ }^{2}$ is set equal to one and $\sigma_{\delta}{ }^{2}$ is allowed to take on the values $\frac{1}{8}, \frac{1}{4}, \frac{1}{2}, 1,2,4$, and 8 . In addition, the $\mu_{i j}$ 's can be set equal to zero without any loss of generality. One thousand data sets were generated for each $\sigma_{\delta}{ }^{2}$ for each of the experimental designs considered, as was done by Remmenga and Johnson.

It is important to note that sometimes the method of moments estimate for $\sigma_{\delta}{ }^{2}$, used in Procedures 1 and 3, is negative which is not useful in practice. The strategy recommended by Remmenga and Johnson is to replace the estimate of $\sigma_{\delta}{ }^{2}$ with zero. For Procedure 2, where minimal sufficient statistics were used instead of the usual sum of squares, Remmenga and Johnson recommended a similar approach where the estimate of $\sigma_{\delta}^{2}$ would otherwise be negative. The REML estimates of $\sigma_{\varepsilon}{ }^{2}$ and $\sigma_{\delta}{ }^{2}$ used in Procedure 4 will always be non-negative. The simulations for this procedure used the $\mathrm{SAS} \otimes$ MIXED procedure with the $\mathrm{ddfm}=\mathrm{kr}$ option in the model statement.

\subsection{SMALL SAMPLE SIMULATION}

The design for the first set of simulations was taken from Example 28.2 of Milliken and Johnson (1994). The model is given by $y_{i j k}=\mu_{i j}+\delta_{k(i)}+\varepsilon_{i j k}$ for $i=1,2, j=1,2$, and 
$k(1)=1,2$ and $k(2)=3,4,5$ where $\delta_{k(i)} \sim N\left(0, \sigma_{\delta}{ }^{2}\right)$ and $\varepsilon_{i j k} \sim N\left(0, \sigma_{\varepsilon}{ }^{2}\right)$ and are distributed independently. The design for this two-way treatment structure is shown in Table 1, where " $\mathrm{x}$ " represents known data and "-" represents missing data. The model can be described with matrix notation as $\mathbf{y}=\mathbf{X} \boldsymbol{\beta}+\mathbf{Z} \boldsymbol{\delta}+\boldsymbol{\varepsilon}$, that is,

$$
\left[\begin{array}{l}
\mathrm{y}_{111} \\
\mathrm{y}_{121} \\
\mathrm{y}_{112} \\
\mathrm{y}_{213} \\
\mathrm{y}_{223} \\
\mathrm{y}_{214} \\
\mathrm{y}_{215} \\
\mathrm{y}_{225}
\end{array}\right]=\left[\begin{array}{llll}
1 & 0 & 0 & 0 \\
0 & 1 & 0 & 0 \\
1 & 0 & 0 & 0 \\
0 & 0 & 1 & 0 \\
0 & 0 & 0 & 1 \\
0 & 0 & 1 & 0 \\
0 & 0 & 1 & 0 \\
0 & 0 & 0 & 1
\end{array}\right] \cdot\left[\begin{array}{l}
\mu_{11} \\
\mu_{12} \\
\mu_{21} \\
\mu_{22}
\end{array}\right]+\left[\begin{array}{lllll}
1 & 0 & 0 & 0 & 0 \\
1 & 0 & 0 & 0 & 0 \\
0 & 1 & 0 & 0 & 0 \\
0 & 0 & 1 & 0 & 0 \\
0 & 0 & 1 & 0 & 0 \\
0 & 0 & 0 & 1 & 0 \\
0 & 0 & 0 & 0 & 1 \\
0 & 0 & 0 & 0 & 1
\end{array}\right] \cdot\left[\begin{array}{l}
\delta_{1(1)} \\
\delta_{2(1)} \\
\delta_{3(2)} \\
\delta_{4(2)} \\
\delta_{5(2)}
\end{array}\right]+\left[\begin{array}{l}
\varepsilon_{111} \\
\varepsilon_{121} \\
\varepsilon_{112} \\
\varepsilon_{213} \\
\varepsilon_{223} \\
\varepsilon_{214} \\
\varepsilon_{215} \\
\varepsilon_{225}
\end{array}\right] .
$$

As noted previously the simulations took all $\mu_{i j}=0$ without loss of generality.

The estimable parameter functions listed in Table 2 were chosen by Remmenga and Johnson because they are the functions (i.e. contrasts) whose estimates are affected by the missing observations. Confidence intervals were constructed using an alpha value of 0.05 and the average confidence interval widths were computed for each of the eight parameter functions. Also, the observed significance levels for testing $\boldsymbol{I}^{\prime} \boldsymbol{\beta}=0$ were recorded for each function.

Table 2 also lists the variance associated with the estimate of each parameter function using Method 1. Consider, for example, the parameter function $\bar{\mu}_{1 .}-\bar{\mu}_{2}$. That is, let $\boldsymbol{I}^{\prime}=\left[\begin{array}{llll}\frac{1}{2} & \frac{1}{2} & -\frac{1}{2} & -\frac{1}{2}\end{array}\right]$. Recall that for Procedure 1 the elements in $\mathbf{b}$ are chosen so that it treats the $\delta_{k(i)}$ equally for every $k$ within each $i$. That is, in the vector $\mathbf{b}^{\prime} \hat{\boldsymbol{\delta}}$ each of $\delta_{1(1)}$ and $\delta_{2(1)}$ receive equal weights of $1 / 2$ while $\delta_{3(2)}, \delta_{4(2)}$, and $\delta_{5(2)}$ receive equal weights of $1 / 3$. Thus, to estimate $\bar{\mu}_{1 .}-\bar{\mu}_{2 .}$, one can take $\mathbf{b}^{\prime}=\left[\begin{array}{lllll}\frac{1}{2} & \frac{1}{2} & -\frac{1}{3} & -\frac{1}{3} & -\frac{1}{3}\end{array}\right]$. Then

$\left[\begin{array}{l}\boldsymbol{l} \\ \mathbf{b}\end{array}\right]^{\prime}\left[\begin{array}{l}\hat{\boldsymbol{\beta}} \\ \hat{\boldsymbol{\delta}}\end{array}\right]=\boldsymbol{I}^{\prime} \hat{\boldsymbol{\beta}}+\mathbf{b}^{\prime} \hat{\boldsymbol{\delta}}=\frac{\hat{\mu}_{11}+\hat{\mu}_{12}}{2}-\frac{\hat{\mu}_{21}+\hat{\mu}_{22}}{2}+\frac{\hat{\delta}_{1(1)}+\hat{\delta}_{2(1)}}{2}-\frac{\hat{\delta}_{3(2)}+\hat{\delta}_{4(2)}+\hat{\delta}_{5(2)}}{3}$

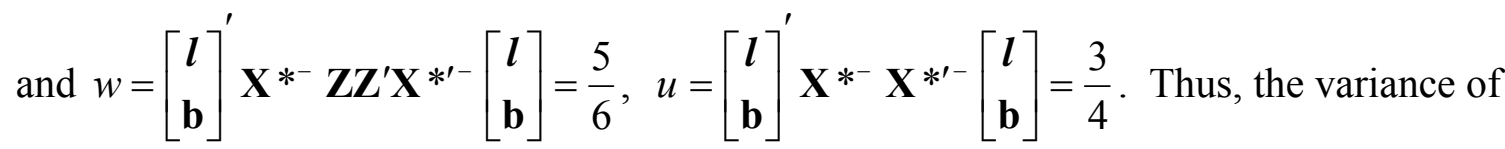
$\boldsymbol{I}^{\prime} \hat{\boldsymbol{\beta}}+\mathbf{b}^{\prime} \hat{\boldsymbol{\delta}}$ is $\operatorname{var}\left[\boldsymbol{l}^{\prime} \hat{\boldsymbol{\beta}}+\mathbf{b}^{\prime} \hat{\boldsymbol{\delta}}\right]=\frac{3}{4} \sigma_{\varepsilon}^{2}+\frac{5}{6} \sigma_{\delta}^{2}=\frac{3}{4}\left(\sigma_{\varepsilon}^{2}+\frac{10}{9} \sigma_{\delta}^{2}\right)$. 
Table 1 Design for Small Sample Experiment

\begin{tabular}{cccc} 
& & \multicolumn{3}{c}{ Week } \\
\cline { 3 - 4 } Subject & Drug & 1 & 2 \\
\hline 1 & 1 & $\mathrm{X}$ & $\mathrm{x}$ \\
2 & 1 & $\mathrm{x}$ & - \\
3 & 2 & $\mathrm{x}$ & $\mathrm{x}$ \\
4 & 2 & $\mathrm{x}$ & - \\
5 & 2 & $\mathrm{x}$ & $\mathrm{x}$
\end{tabular}

Table 2 Parameter functions and the variances of their estimates for the Small Sample Experiment

\begin{tabular}{|c|c|}
\hline$l^{\prime} \beta$ & $\operatorname{var}\left[\boldsymbol{I}^{\prime} \hat{\boldsymbol{\beta}}+\mathbf{b}^{\prime} \hat{\boldsymbol{\delta}}\right]=u\left(\sigma_{\varepsilon}^{2}+\frac{w}{u} \sigma_{\delta}^{2}\right)$ \\
\hline$\mu_{12}$ & $3 / 2\left(\sigma_{\varepsilon}^{2}+1 / 3 \sigma_{\delta}^{2}\right)$ \\
\hline$\mu_{22}$ & $2 / 3\left({\sigma_{\varepsilon}}^{2}+1 / 2{\sigma_{\delta}}^{2}\right)$ \\
\hline$\mu_{12}-\mu_{22}$ & $3 / 6\left(\sigma_{\varepsilon}^{2}+5 / 13{\sigma_{\delta}}^{2}\right)$ \\
\hline $\bar{\mu}_{\cdot 2}$ & $13 / 24\left({\sigma_{\varepsilon}}^{2}+5 / 13{\sigma_{\delta}}^{2}\right)$ \\
\hline $\bar{\mu}_{2}$ & $1 / 4\left(\sigma_{\varepsilon}^{2}+4 / 3 \sigma_{\delta}^{2}\right)$ \\
\hline $\bar{\mu}_{1 .}-\bar{\mu}_{2}$ & $3 / 4\left({\sigma_{\varepsilon}}^{2}+10 / 9{\sigma_{\delta}}^{2}\right)$ \\
\hline $\bar{\mu}_{\cdot 1}-\bar{\mu}_{\cdot 2}$ & $3 / 4\left(\sigma_{\varepsilon}^{2}\right)$ \\
\hline$\mu_{11}-\mu_{12}-\mu_{21}+\mu_{22}$ & $3\left(\sigma_{\varepsilon}^{2}\right)$ \\
\hline
\end{tabular}

The results of the simulations for computing confidence intervals for $\mu_{12}-\mu_{22}$ are shown in Figure 1. The abscissa has the values $\log _{2}\left(\sigma_{\delta}^{2}\right)$. The observed confidence levels and confidence interval widths are both plotted where the ordinates on the left give the observed confidence interval widths and the ordinates on the right give the observed confidence levels.

First, note that all of the procedures show acceptable confidence levels with confidence levels varying between $92.3 \%$ and $98.5 \%$ for all estimable functions considered in the small sample simulations and between $94.3 \%$ and $95.7 \%$ for the function $\mu_{12}-\mu_{22}$. Notice that for the small sample simulations the MSS method seems to give the highest confidence levels. However, the confidence interval widths are extremely large which is not useful. The REML estimates seem to give the lowest confidence levels and the lowest confidence interval widths. Procedures 1 and 3 are in between these two procedures. Similar results are obtained for each of the other functions for the fixed effect parameters that were considered and for the significance levels for testing $\boldsymbol{I}^{\prime} \boldsymbol{\beta}=0$. See Smith (2003) for additional details. 
Figure 1 Plot of $\mu_{12}-\mu_{22}$ from the Small Sample Simulations

\section{Parameter Function: $\mu_{12}-\mu_{2} z$}

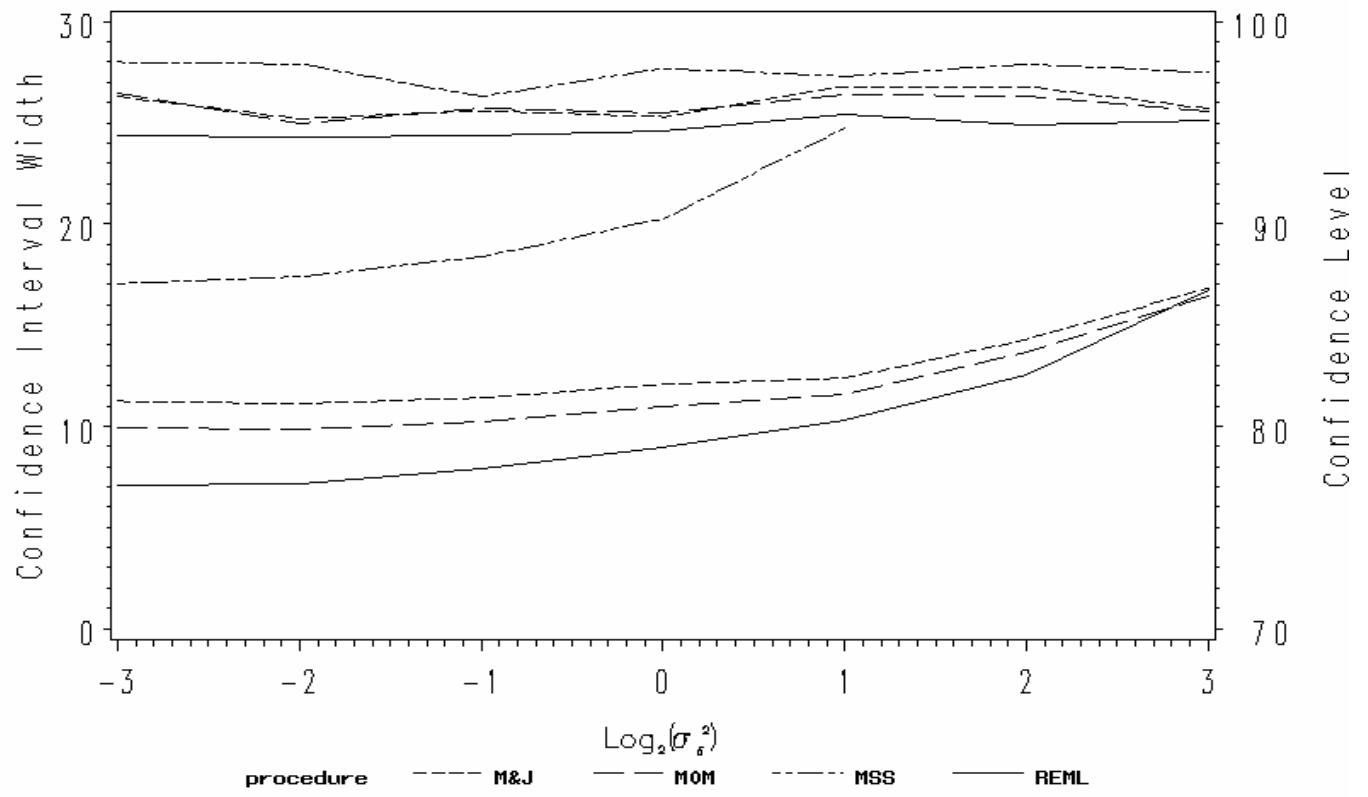

\subsection{LARGE SAMPLE SIMULATION}

The design for this simulation was motivated by Example 28.1 of Milliken and Johnson (1994). The model is given by $y_{i j k}=\mu_{i j}+\delta_{k(i)}+\varepsilon_{i j k}$ for $i=1,2,3, j=1,2,3,4$ and $k(1)=1,2$, $3, k(2)=4,5,6,7,8$ and $\mathrm{k}(3)=9,10,11,12$. Again, without loss of generality, let $\mu_{i j}=0$ for all $i \neq j$. The example from Milliken and Johnson only has missing whole plot treatments. This paper considers, as did Remmenga and Johnson, this experiment and three other designs based on this experiment where subplot observations are also missing. The second design was formed by randomly removing approximately $10 \%$ of the observations from the original design, the third by randomly removing another $10 \%$ of the observations. The fourth design has approximately $30 \%$ of the observations removed from the original design. All of the designs for these two-way experiments are shown in Table 3, as they were first described by Remmenga and Johnson.

Table 4 lists the twelve estimable parameter functions that were considered by Remmenga and Johnson. These functions were selected because they are functions whose estimates are most likely to be affected by the unbalanced designs. An additional parameter function comparing two whole plot treatment main effect means was also included. Remmenga and Johnson listed the values for $q, u$, and $v$ but they are not be reprinted here. Confidence intervals were constructed using an alpha value of 0.05 and the average confidence interval widths were computed for each of twelve parameter functions. Also, the observed significance levels for testing $\boldsymbol{I} \boldsymbol{\beta} \boldsymbol{\beta}=0$ were recorded for each function. 
Table 3 Diagram of experimental designs used in the large sample simulations.

\begin{tabular}{cccccc}
\hline \multirow{2}{*}{$\begin{array}{c}\text { Experimental } \\
\text { unit }\end{array}$} & $\begin{array}{c}\text { Whole plot } \\
\text { treatment }\end{array}$ & \multicolumn{4}{c}{ Subplot treatment } \\
\cline { 3 - 6 } & $\mathrm{A}$ & $\mathrm{iii}$ & $\mathrm{X}$ & $\mathrm{X}$ & $\mathrm{X}$ \\
2 & $\mathrm{~A}$ & $\mathrm{X}$ & iii & $\mathrm{X}$ & $\mathrm{i}$ \\
3 & $\mathrm{~A}$ & $\mathrm{ii}$ & $\mathrm{X}$ & $\mathrm{i}$ & $\mathrm{X}$ \\
4 & $\mathrm{~B}$ & $\mathrm{X}$ & $\mathrm{ii}$ & $\mathrm{X}$ & $\mathrm{X}$ \\
5 & $\mathrm{~B}$ & $\mathrm{X}$ & $\mathrm{X}$ & $\mathrm{X}$ & $\mathrm{X}$ \\
6 & $\mathrm{~B}$ & $\mathrm{X}$ & $\mathrm{X}$ & $\mathrm{iii}$ & $\mathrm{X}$ \\
7 & $\mathrm{~B}$ & $\mathrm{iii}$ & $\mathrm{X}$ & $\mathrm{X}$ & $\mathrm{X}$ \\
8 & $\mathrm{~B}$ & $\mathrm{i}$ & ii & $\mathrm{X}$ & $\mathrm{X}$ \\
9 & $\mathrm{C}$ & $\mathrm{X}$ & $\mathrm{X}$ & $\mathrm{X}$ & $\mathrm{X}$ \\
10 & $\mathrm{C}$ & $\mathrm{X}$ & ii & $\mathrm{X}$ & $\mathrm{X}$ \\
11 & $\mathrm{C}$ & $\mathrm{i}$ & $\mathrm{X}$ & ii & $\mathrm{X}$ \\
12 & $\mathrm{C}$ & $\mathrm{X}$ & $\mathrm{X}$ & $\mathrm{X}$ & $\mathrm{X}$ \\
\hline
\end{tabular}

i - Removed from Design 1 to create Design 2.

ii - Removed from Design 2 to create Design 3.

iii - Removed from Design 3 to create Design 4.

Table 4 Parameter functions for the Large Sample Simulations

$\begin{array}{llll}\mu_{11} & \bar{\mu}_{2 .} & \mu_{13}-\mu_{33} & \\ \mu_{21} & \bar{\mu}_{\cdot 1}-\bar{\mu}_{\cdot 2} & \mu_{14}-\mu_{34} & \\ \mu_{31} & \mu_{11}-\mu_{21} & \mu_{11}-\mu_{14} & \\ \mu_{12}-\mu_{22} & \mu_{12}-\mu_{22} & \bar{\mu}_{1 .}-\bar{\mu}_{2 .} \quad \text { (additional function) }\end{array}$

Figures 2 and 3 contain the simulation results for confidence intervals for $\mu_{11}-\mu_{21}$ for the large sample simulations of designs 1 and 4 , respectively. The abscissa again has the values $\log _{2}\left(\sigma_{\delta}^{2}\right)$. The observed confidence levels and confidence interval widths are both plotted on the same graphs where the left ordinates correspond to the observed confidence interval widths and the right ordinates correspond to the observed confidence levels.

First, note that all of the procedures show acceptable confidence interval widths and confidence levels with observed confidence levels varying between $92.0 \%$ and $99.2 \%$ for all estimable functions considered in the large sample simulations. For Design 1 the confidences levels for $\mu_{11}-\mu_{21}$ are between $93.3 \%$ and $95.7 \%$ and between $93.9 \%$ and $95.6 \%$ for Design 4 . 
Figure 2 Plot of $\mu_{11}-\mu_{21}$ from the Large Sample Simulations of Design 1

Parameter Function: $\mu_{\mathbf{1}}-\mu_{\mathbf{2}} \mathbf{1}$

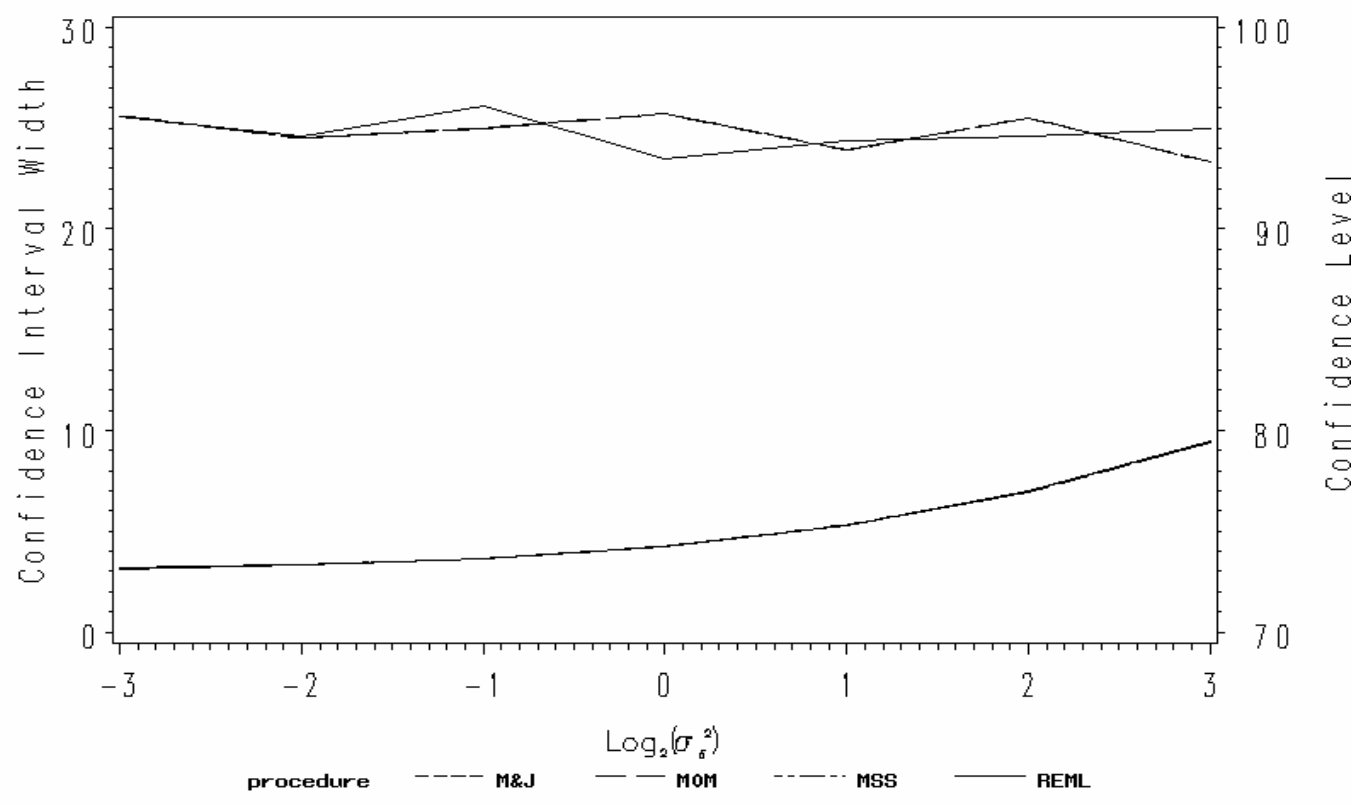

Figure 3 Plot of $\mu_{11}-\mu_{21}$ from the Large Sample Simulations of Design 4

Parameter Function: $\mu_{\mathbf{1}}-\mu \mathbf{z}$

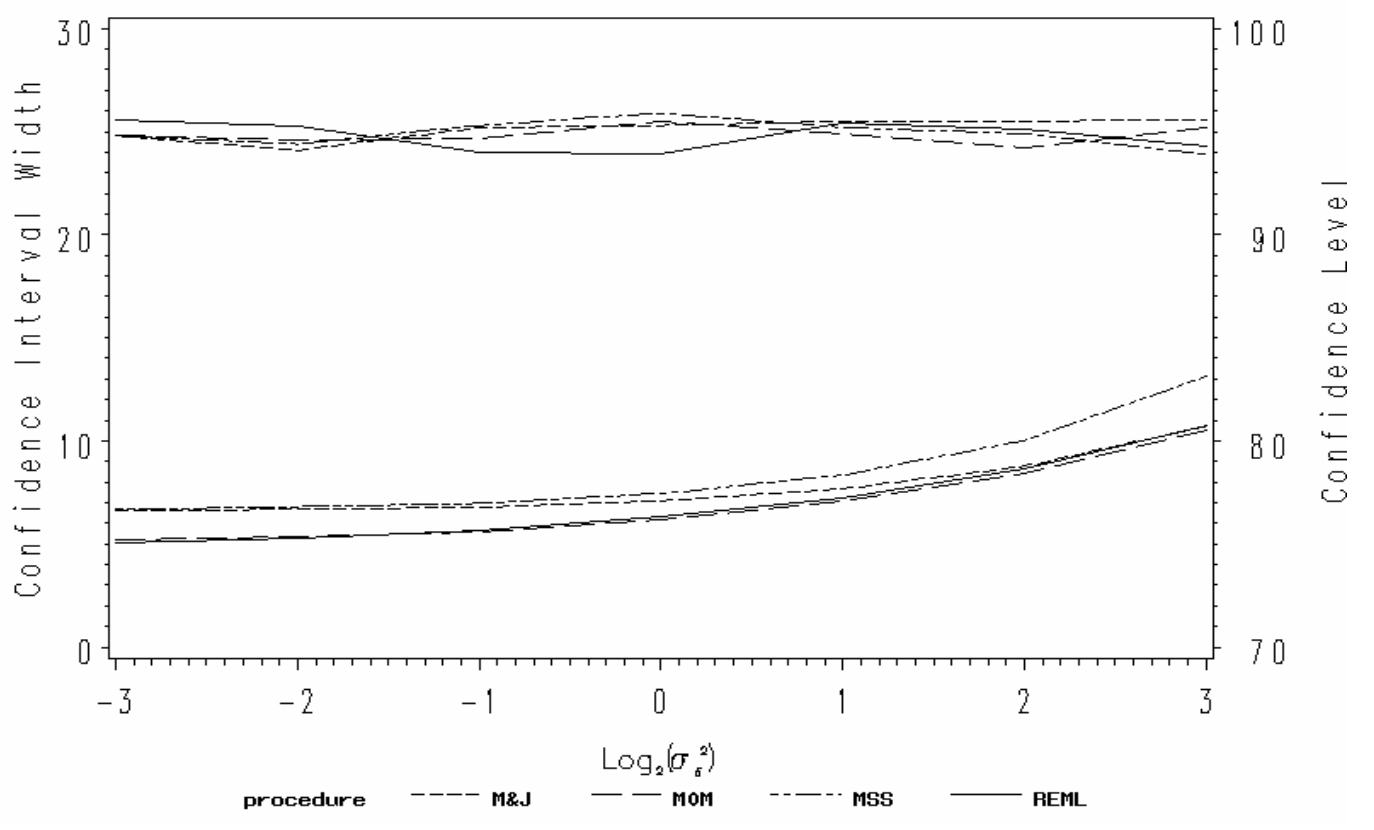


In Design 1 the three procedures recommended by Remmenga and Johnson behave exactly the same and the REML procedure behaves only slightly different. All four procedures have the same confidence interval width for Design 1. Confidence levels and confidence interval widths are acceptable for all four procedures in Design 4. No procedure seems to always have the highest confidence level. However, the minimal sufficient statistics do seem to have larger confidence interval widths for Design 4. Similar results were observed for each of the other functions of the fixed effect parameters that were considered for the significance levels for testing $\boldsymbol{I}^{\prime} \boldsymbol{\beta}=0$. See Smith (2003) for additional details.

It should also be noted that, the REML estimate of $\sigma_{\delta}{ }^{2}$ is equal to zero whenever the method of moments estimate of $\sigma_{\delta}{ }^{2}$ is negative. Remmenga and Johnson (1995, Table 5) reported that negative method of moment estimates were rarely encountered as long as $\frac{\sigma_{\delta}^{2}}{\sigma_{\varepsilon}^{2}} \geq 1$. When $\frac{\sigma_{\delta}^{2}}{\sigma_{\varepsilon}^{2}}=\frac{1}{2}$, the probability of negative method of moment estimates ranged from 0.040 to 0.123 for the four designs considered. When $\frac{\sigma_{\delta}^{2}}{\sigma_{\varepsilon}^{2}}=\frac{1}{4}$, the probabilities ranged from 0.114 to 0.237 , and when $\frac{\sigma_{\delta}^{2}}{\sigma_{\varepsilon}^{2}}=\frac{1}{8}$, the probabilities ranged from 0.040 to 0.123

\section{CONCLUSIONS}

Four procedures for constructing confidence intervals and testing hypotheses about fixed effects in unbalanced split-plot experiments have been presented and discussed. First, it should be noted that the three procedures recommended by Remmenga and Johnson were recommended because they behaved well. That is they had acceptable confidence levels for nearly all values of $\sigma_{\delta}{ }^{2}$, with observed confidence levels greater than $93 \%$ for Procedures 1 and 3 and greater than $92 \%$ for Procedure 2 . They also have reasonable confidence interval widths and test sizes for hypothesis tests of $\boldsymbol{I}^{\prime} \boldsymbol{\beta}=0$. Remmenga and Johnson observed that as the number of missing observations increased, the variation of the confidence levels given by Procedure 3 increases. Procedure 4 performs as well as the three procedures recommended by Remmenga and Johnson. It gave acceptable confidence levels, nearly all being above $93 \%$, and reasonable confidence interval widths and hypothesis test sizes.

For the small data sets, Procedure 2 generally has the highest confidence level but the confidence interval width is also quite large. Procedure 4 has the smallest confidence level and the smallest confidence interval width. Procedure 4 tends to have the largest test size and Procedure 2 tends to have the smallest test size for the small data set (Smith, 2003). Procedures 1 and 3 behave similarly to one another and reasonably for confidence level, confidence interval width and hypothesis test size.

When the larger data sets were considered all four procedures were nearly indistinguishable for confidence level, confidence interval width and hypothesis test size where 
there was little missing data. As the amount of missing data increases the behavior of all four procedures becomes more varied. Procedure 4 seems to be less erratic than the other three procedures but never has consistently higher or lower confidence levels. However, Procedure 4 does tend to have the smallest confidence interval widths.

In general, for designs 1-4 all four procedures perform similarly and no procedure stands out as being consistently better than the others. However, Procedure 2 tends to have the worst behavior. All of the procedures behave more erratically at larger values of $\sigma_{\delta}^{2}$ as should be expected.

Based on the results of the simulation study, it appears that SAS ${ }^{\circledR}$ MIXED procedure with its $\mathrm{METHOD}=\mathrm{REML}$ and $\mathrm{ddfm}=\mathrm{kr}$ options is an appropriate procedure for analyzing unbalanced split-plot experiments. It is also reasonable to assume that these results can be extended to experimental designs that include multiple splits or strips.

\section{REFERNCES}

1. Hultquist, Robert and Erwind Atzinger. (1972). "The Mixed Effects Model and Simultaneous Diagonalization of Symmetric Matrices." The Annals of Mathematical Statistics, 43, 2024-2030.

2. Huynh, Huynh and Feldt, Leonard (1970). "Conditions Under Which Mean Squared Ratios in Repeated Measurements Designs Have Exact F-Distributions." Journal of the American Statistical Association, 65, 1582-1589.

3. Kackar, Raghu N. and David A. Harville (1984). "Approximations for Standard Error of Estimators of Fixed and Random Effects in Mixed Linear Model." Journal of the American Statistical Association, 79, 853-862.

4. Kenward, Michael G. and James H. Roger. (1997). "Small Sample Inferance for Fixed Effects from Restricted Maximum Likelihood.” Biometrics, 53, 983-997.

5. McLean, Robeer A. and William L. Sanders. (1988). "Approximating Degrees of Freedom for Standard Errors in Mixed Linear Models." Proceedings of the Statistics Computing Section. American Statistical Association, New Orleans: 50-59.

6. Milliken, G. A. and D. E. Johnson. (1994). Analysis of Messy Data Vol. 1: Designed Experiments. Chapman \& Hall. New York.

7. Remmenga, Marta D. (1992). "On Inference Procedures in Unbalanced Split-Plot Designs." Unpublished Ph.D. dissertation, Department of Statistics, Kansas State University, Manhattan, KS.

8. Remmenga, Marta D. and Dallas E. Johnson. (1995). "A Comparison of Inference Procedures in Unbalanced Split-plot Designs." J. Statistical Computation and Simulation, 51, 353-367.

9. Smith, Christina D. (2003). "Comparing the Analyses of Unbalance Split-plot Experiments using Mixed Models with REML Estimates of the Variance Components to other Inference Procedures Previously Recommended." Unpublished Masters thesis, Department of Statistics, Kansas State University, Manhattan, KS. 